\title{
Prevalence and determinants of unintended pregnancy among women in Nairobi, Kenya
}

\author{
Lawrence Ikamari ${ }^{*}$, Chimaraoke Izugbara ${ }^{2}$ and Rhoune Ochako ${ }^{3}$
}

\begin{abstract}
Background: The prevalence of unintended pregnancy in Kenya continues to be high. The 2003 Kenya Demographic and Health Survey (KDHS) showed that nearly 50\% of unmarried women aged $15-19$ and $45 \%$ of the married women reported their current pregnancies as mistimed or unwanted. The 2008-09 KDHS showed that 43\% of married women in Kenya reported their current pregnancies were unintended. Unintended pregnancy is one of the most critical factors contributing to schoolgirl drop out in Kenya. Up to 13,000 Kenyan girls drop out of school every year as a result of unintended pregnancy. Unsafe pregnancy termination contributes immensely to maternal mortality which currently estimated at 488 deaths per 100000 live births. In Kenya, the determinants of prevalence and determinants of unintended pregnancy among women in diverse social and economic situations, particularly in urban areas, are poorly understood due to lack of data. This paper addresses the prevalence and the determinants of unintended pregnancy among women in slum and non-slum settlements of Nairobi.
\end{abstract}

Methods: This study used the data that was collected among a random sample of 1262 slum and non-slum women aged 15-49 years in Nairobi. The data was analyzed using simple percentages and logistic regression.

Results: The study found that 24 percent of all the women had unintended pregnancy. The prevalence of unintended pregnancy was 21 per cent among women in slum settlements compared to 27 per cent among those in non-slum settlements. Marital status, employment status, ethnicity and type of settlement were significantly associated with unintended pregnancy. Logistic analysis results indicate that age, marital status and type of settlement had statistically significantly effects on unintended pregnancy. Young women aged 15-19 were significantly more likely than older women to experience unintended pregnancy. Similarly, unmarried women showed elevated risk for unintended pregnancy than ever-married women. Women in non-slum settlements were significantly more likely to experience unintended pregnancy than their counterparts in slum settlements.

The determinants of unintended pregnancy differed between women in each type of settlement. Among slum women, age, parity and marital status each had significant net effect on unintended pregnancy. But for non-slum women, it was marital status and ethnicity that had significant net effects.

Conclusion: The study found a high prevalence of unintended pregnancy among the study population and indicated that young and unmarried women, irrespective of their educational attainment and household wealth status, have a higher likelihood of experiencing unintended pregnancy. Except for the results on educational attainments and household wealth, these results compared well with the results reported in the literature. The results indicate the need for effective programs and strategies to increase access to contraceptive services and related education, information and communication among the study population, particularly among the young and unmarried women. Increased access to family planning services is key to reducing unintended pregnancy among the study population. This calls for concerted efforts by all the stakeholders to improve access to family planning (Continued on next page)

\footnotetext{
*Correspondence: likamari@uonbi.ac.ke

${ }^{1}$ Population Studies and Research Institute, University of Nairobi, P.O.BOX 30197, 00100, GPO, Nairobi, Kenya

Full list of author information is available at the end of the article
}

\section{Biomed Central}

(c) 2013 Ikamari et al.; licensee BioMed Central Ltd. This is an Open Access article distributed under the terms of the Creative Commons Attribution License (http://creativecommons.org/licenses/by/2.0), which permits unrestricted use, distribution, and reproduction in any medium, provided the original work is properly cited. 
services among the study population. Increased access should be accompanied with improvement in the quality of care and availability of information about effective utilization of family planning methods.

Keywords: Unintended pregnancy, Determinants, Slum, Non-slum settlements, Urban, Nairobi, Kenya

\section{Background}

Unintended pregnancy, which includes both mistimed and unwanted pregnancies, is a global social and health challenge. Worldwide, $38 \%$ of pregnancies are unintended (that is, some 80 million pregnancies annually). In subSaharan Africa, unintended pregnancy accounts for more than a quarter of the 40 million pregnancies that occur annually. Unintended pregnancies increase health and economic risks for children, women, men and families. Research indicates that unintentional pregnancy is a key risk factor for adverse pregnancy and maternal outcomes, including mortality and morbidity associated with unsafe induced abortions [1-3] Unintended pregnancy has also been linked to low use of appropriate maternal health care $[2,4] ;[5,6]$. Unintended pregnancy is also a major cause of unsafe abortion [1-3,6].

As in most of Africa, the prevalence of unintended pregnancy in Kenya continues to be high. In Adetunji's [7] study of eight sub-Saharan African countries, Kenya recorded the highest proportion of unintended childbearing. In the 2003, the Kenya Demographic and Health Survey (KDHS) showed that nearly $50 \%$ of unmarried women aged $15-19$ and $45 \%$ of the married women reported their current pregnancies as mistimed or unwanted [8]. The 2008-09 KDHS showed that 43\% (26\% mistimed and 17\% unwanted) of married women in Kenya reported their current pregnancies as unintended [9]. Unintended pregnancy is one of the most critical factors contributing to schoolgirl drop out in Kenya. Up to 13,000 Kenyan girls drop out of school every year as a result of unintended pregnancy [10] In addition, unsafe pregnancy termination contributes immensely to maternal mortality which currently estimated at 488 deaths per 100000 live births [9].

Studies have shown a wide range of correlates of unintended pregnancy. Unintended pregnancies mostly arise as a result of nonuse or incorrect use of contraceptives, or a noticeable contraceptive failure $[6,7,11]$. Unintended pregnancies have also been shown to be strongly associated with maternal age and number of previous births [2,7,11-13]. A prospective study in 2 governorates of Upper Egypt revealed that the majority of women never used contraception, and unintended pregnancy was more prevalent in this category of women compared to those who had ever contraception used [14].

In Chile, women aged less than 25 and of low socioeconomic status were more likely, than their peers living in households of better socioeconomic status, to have unplanned pregnancies [15]. In Harare, a significant association was found between unintended pregnancy and age, with women aged 19 years and below or 35 years and above having a higher risk of unintended pregnancy [16]. Similar results have been reported in several other studies. Young women have higher likelihood of inconsistent or nonuse of effective family planning methods than older women and have greater risk to have mistimed than intended pregnancy [17-19]. Urban women, furthermore, are less likely than rural women to have more children than that which they regard as ideal. Research from different countries also indicate that women with better education levels were less likely than those with less education levels to have more children than that which they regard as ideal. Moreover, the higher education and the better socioeconomic status a woman had, and then it is less likely for her to have an unplanned pregnancy [2,7,11-13].

Existing literature on unintended pregnancy in Kenya has addressed its socio-demographic correlates, national prevalence, implications for maternal and child health and care-seeking, and repeatability $[5,7,20]$. These studies have relied largely on national large-scale or localized facility-based surveys. Little is therefore known about the prevalence and determinants of pregnancy among women from diverse socio-economic and livelihoods, particularly in urban areas of Kenya. The current study addresses the prevalence and the determinants of unintended pregnancy among women in slum and non-slum settlements of Nairobi.

Following rapid urban growth under enormous economic constraints, an increasing proportion of Kenyans now live in cities. However, urbanization in Kenya has produced critical geographic concentrations characterized by both prosperity and poverty. Cities, deeply divided along socio-economic lines, have thus emerged all over the country. Currently, high-rent neighborhoods characterized by affluence exist next to slums noted for their squalor and impoverished livelihoods. Generally, livelihood conditions vary clearly between these zones, often translating into objective differences in health outcomes [21].

Poor urban settlement contexts set limits on the ability of women and men to safeguard their sexual and reproductive health, control their fertility, and implement their fertility aspirations [21,22]. Essentially, these settlements are characterized by extreme poverty and poor livelihood conditions, limited access to family planning 
services, illiteracy, sexual violence, and lack of access to quality health care, including ante and post-natal care services. They present particularly interesting and fertile locations for unintended pregnancy and related behavior [21].

\section{Study objectives}

The goal of this study was to generate new knowledge on the prevalence and determinants of unintended pregnancy among slum and non-slum women in Nairobi, Kenya. Specifically, the study sought to (a): examine the prevalence of unintended pregnancy in study settlements and (b): explore the socio economic and demographic determinants of unintended pregnancy in the study communities.

\section{Method}

\section{Source of data}

The data for this paper were drawn from the study on "Prevalence, Perceptions, and Experiences of Unwanted Pregnancy among women in slum and non-slum settlements of Nairobi, Kenya" conducted by the African Population and Health Research Centre (APHRC) in 2009-10. The study was conducted among women aged 15-49 years in four communities- Korogocho, Viwandani, Jericho, and Harambee in Nairobi. Korogocho and Viwandani are slum settlements whereas Jericho and Harambeeare nonslum Settlements. The study collected data from a total of 1962 randomly-selected women. A two-stage sampling design was employed to recruit study participants. The initial stage involved a random sampling of households from the settlements.

The sample of households was drawn from APHRC's Nairobi Urban Health and Demographic Surveillance System (NUHDSS) which is implemented in these settlements. The second stage involved a simple random selection of one eligible woman in each of the sampled households. In the study, information was collected on women's social, economic, demographic, pregnancy, birth histories (including miscarriages and or abortions, stillbirths, and neonatal deaths) as well as contraceptive behavior. It also collected information on unintended pregnancy among women, the number of times this had happened, and why the pregnancy was considered unintended. Women who admitted to experiencing unintended pregnancy were also asked how they managed the pregnancy. This paper is based on 1,272 women who rereported ever being pregnant and who indicated whether their most recent pregnancy was intended or not.

The study was approved by the Kenya Medical Research Institute (KEMRI). Informed consent for participation was also obtained from each of the respondents.

\section{Study variables}

The dependent variable is pregnancy intention, measured as a two-outcome variable and coded as intended pregnancy, if the pregnancy occurred at a time when the woman wanted it, and unintended pregnancy, if the pregnancy occurred at a time when the woman would have wanted it later or did not want it at all. The independent variables used in this paper include education (coded as none, primary and secondary/higher), wealth index (recoded as tertiles and labeled poor, middle and rich), ethnicity, parity, age, marital status, household size, employment status, and type of residence. These are some of the variables that have been found to affect incidence of unintended pregnancy elsewhere.

\section{Data analysis}

The study used a mix of methods for data analysis. Simple percentages and cross-tabulation are used to analyze the levels and differentials in unintended pregnancy. Logistic regression is used in multivariate analysis of factors affecting unintended pregnancy. Results are presented as risk ratios, which represent the relative likelihood of exposure to the variable of interest. The risk ratio of the reference group or category is one (1.00). An odds ratio of greater than 1.00 indicates increased likelihood of experiencing unintended pregnancy while an odds ratio of less than 1.00 indicates a lower likelihood of experiencing unintended pregnancy. In the study, independent variables are considered significant if their effects on unintended pregnancy are statistically significant at the 95 per cent level of significance.

\section{Results}

Basic socio-economic and demographic characteristics of the study population

Table 1 shows the socio-demographic characteristics of 1,272 women who were ever pregnant and reported whether their last pregnancy was intended or unintended. Slightly more than half $(58 \%)$ of the women were from slum settlements, majority of the women $(60 \%)$, were aged 20-34 years while $43 \%$ had primary level education. More than half of the women were $62 \%$ currently married and majority of the households (54\%) had between 3 and 5 persons while $59 \%$ of the women were of parity 1 and 2 . Considering ethnic affiliation, Kikuyu women were the majority (34\%). Twenty-four percent of the pregnancy occurring among these women was reported as unintended, meaning they occurred at a time when the woman would have preferred to have it later or did not want it at all.

\section{Prevalence of unintended pregnancy}

About $24 \%$ of the 1272 women had unintended pregnancy. The results show statistically significant variation in the incidence of unintended pregnancy according to the number of characteristics. Never married women were more likely to experience unintended pregnancy; 
Table 1 Percentage distribution of variables in the sample included in the analysis among women aged 15-49 years

\begin{tabular}{lcc}
\hline Characteristics & Frequency & Percent \\
\hline Age & & \\
Less than 20 & 19 & 1.5 \\
$20-34$ & 760 & 59.7 \\
$35-49$ & 493 & 38.8
\end{tabular}

Level of education

None

Primary

Secondary

Higher

Marital status

Never Married

Currently Married

Formerly married

Household size

1-2 persons

3-5 persons

6 and above

Parity

0

1-2 births

3 and above births

Wealth index

Poor

Medium

Rich

Type of residence

Slum

Non-slum

Ethnicity

Kikuyu

Luhya

Luo

Kamba

Other

Employment status

Unemployed/student

Informal employment

Formal employment

Self employed

Pregnancy intention

Intended pregnancy

Unintended pregnancy

Total
$62 \%$ of them reported having had unintended pregnancy compared to $13 \%$ and $26 \%$ of currently and formerly married women respectively. Women who were either unemployed or students and those in informal employment had a prevalence of $28 \%$. Women in formal employment and those in self-employment had lower incidence of unintended pregnancy $(17.5 \%$ and $22 \%$ respectively). Zero parity women had the highest prevalence of experiencing unintended pregnancy, 30\% compared to $27 \%$ and $20 \%$ among those of parity $1-2$ and parity 3 and above respectively. Women residing in the non-slum settlements had higher prevalence of unintended pregnancy $(27 \%)$ than their counterparts in the Slum settlements (22\%).

Luhya and Luo women had the highest prevalence of unintended pregnancy at $30 \%$ and $34 \%$ respectively. Women aged 15-19 had the highest prevalence of unintended pregnancy, (68\%) while it was least among women aged 35-49 at 20\%. Households with 1-2 persons and those with at least 6 persons experienced the highest prevalence of unintended pregnancy at $29 \%$.

However, the results indicate no statistically significant variation in the incidence of unintended pregnancy according to the woman's education level and the wealth index of her household (Table 2).

\section{Socio-economic and demographic determinants of unintended pregnancy}

The results of the analysis of the determinants of unintended pregnancy among the women who took part in this study are presented in two models. Model I fitted the outcome variable and the socioeconomic variables namely: education, wealth index, employment status, ethnicity, household size and residence. Model II fitted all the variables included in Model I together with age, parity and marital status. The results of the two models are presented in Table 3.

As in the case of the bivariate analysis, the results shown in Model I indicate that education was not statistically associated with the occurrence of unintended pregnancy among the study population. However, household wealth index was closely associated with unintended pregnancy $(\mathrm{p}<0.05)$. Women from medium and rich households were $66 \%(\mathrm{p}<0.05)$ and $51 \%(\mathrm{p}<0.02)$ respectively less likely to experience unintended pregnancy compared to women from poor households. Women in formal employment and those in self-employment were $49 \%(\mathrm{p}<0.001)$ and $72 \%(\mathrm{p}<0.05)$ respectively less likely to experience unintended pregnancy compared to those who were unemployed or students.

The results indicate that the likelihood of experiencing unintended pregnancy was high among Luhya and Luo women with each being 46\% $(\mathrm{p}<0.05)$ and $73 \%(\mathrm{p}<0.005)$ respectively more likely than Kikuyu women. While 
Table 2 Prevalence of unintended pregnancy among the study population, Nairobi, Kenya

\begin{tabular}{|c|c|c|c|}
\hline & $\begin{array}{l}\text { Intended } \\
\text { pregnancy }\end{array}$ & $\begin{array}{r}\text { Unintended } \\
\text { pregnancy }\end{array}$ & Total \\
\hline Education & & $p=0.747$ & \\
\hline None & 83.3 & 16.7 & 100.0 \\
\hline Primary & 76.4 & 23.6 & 100.0 \\
\hline Secondary & 75.3 & 24.7 & 100.0 \\
\hline Higher & 75.7 & 24.3 & 100.0 \\
\hline Marital status & & $p=0.000$ & \\
\hline Never married & 38.2 & 61.8 & 100.0 \\
\hline Currently married & 86.7 & 13.3 & 100.0 \\
\hline Formerly married & 73.8 & 26.2 & 100.0 \\
\hline Wealth index & & $p=0.969$ & \\
\hline Poor & 76.2 & 23.8 & 100.0 \\
\hline Medium & 76.4 & 23.6 & 100.0 \\
\hline Rich & 75.6 & 24.4 & 100.0 \\
\hline Employment status & & $p=0.010$ & \\
\hline Unemployed/student & 72.5 & 27.5 & 100.0 \\
\hline Informal employment & 71.7 & 28.3 & 100.0 \\
\hline Formal employment & 82.5 & 17.5 & 100.0 \\
\hline Self employed & 78.4 & 21.6 & 100.0 \\
\hline Parity & & $p=0.010$ & \\
\hline 0 & 70.0 & 30.0 & 100.0 \\
\hline 1-2 children & 73.3 & 26.7 & 100.0 \\
\hline $3+$ children & 80.5 & 19.5 & 100.0 \\
\hline Residence & & $p=0.017$ & \\
\hline Slum & 78.5 & 21.5 & 100.0 \\
\hline Non-slum & 72.8 & 27.2 & 100.0 \\
\hline Ethnicity & & $p=0.000$ & \\
\hline Kikuyu & 79.5 & 20.5 & 100.0 \\
\hline Luhya & 69.7 & 30.3 & 100.0 \\
\hline Luo & 65.7 & 34.3 & 100.0 \\
\hline Kamba & 80.7 & 19.3 & 100.0 \\
\hline Other & 83.1 & 16.9 & 100.0 \\
\hline Age & & $p=0.000$ & \\
\hline $15-19$ & 31.6 & 68.4 & 100.0 \\
\hline $20-34$ & 74.5 & 25.5 & 100.0 \\
\hline $35-49$ & 80.3 & 19.7 & 100.0 \\
\hline House hold size & & $p=0.000$ & \\
\hline $1-2$ persons & 70.8 & 29.2 & 100.0 \\
\hline 3-5 persons & 80.6 & 19.4 & 100.0 \\
\hline $6+$ persons & 71.0 & 29.0 & 100.0 \\
\hline
\end{tabular}

Table 3 Odds ratio, based on logistic regression analysis, of unintended pregnancy among women, 15-49 in slum and non-settlements in Nairobi Kenya

\begin{tabular}{lccccc}
\hline Characteristic & Model I & & \multicolumn{2}{c}{ Model II } \\
\cline { 2 - 6 } & OR & $\boldsymbol{p}$ & OR & $\boldsymbol{p}$ \\
\hline Education [Ref: None] & 1.43 & 0.454 & 1.24 & 0.659 \\
$\quad$ Primary & 1.32 & 0.562 & 1.11 & 0.830 \\
Secondary & 1.52 & 0.417 & 0.99 & 0.982 \\
Higher & & & & \\
Wealth index [Ref: Poor] & & & & \\
$\quad$ Medium & 0.66 & 0.049 & 0.73 & 0.150 \\
Rich & 0.51 & 0.024 & 0.65 & 0.180
\end{tabular}

Employment status [Ref: Unemployed/student]

$\begin{array}{lllll}\text { Informal employment } & 0.99 & 0.951 & 0.87 & 0.556 \\ \text { Formal employment } & 0.49 & 0.001 & 0.55 & 0.014 \\ \text { Self employed } & 0.72 & 0.045 & 0.80 & 0.237\end{array}$

Ethnicity [Ref: Kikuyu]

$\begin{array}{lllll}\text { Luhya } & 1.46 & 0.052 & 1.80 & 0.007 \\ \text { Luo } & 1.73 & 0.005 & 2.02 & 0.001 \\ \text { Kamba } & 0.91 & 0.664 & 1.18 & 0.460 \\ \text { Other } & 0.75 & 0.253 & 1.20 & 0.744\end{array}$

Household size [Ref: 1-2 persons]

3-5 persons

$\begin{array}{llll}0.50 & 0.002 & 0.76 & 0.282\end{array}$

$6+$ persons

$\begin{array}{llll}0.74 & 0.205 & 0.97 & 0.922\end{array}$

Residence [Ref: Slum]

Non-slum

$\begin{array}{llll}2.20 & 0.002 & 1.80 & 0.034\end{array}$

Age [Ref: 15-19]

20-34

$0.37 \quad 0.081$

$35-49$

$0.35 \quad 0.072$

Parity [Ref: 0]

1-2 children

$1.71 \quad 0.353$

$3+$ children

Marital status [Ref: Never married]

Currently married

$0.09 \quad 0.000$

Formerly married

$0.21 \quad 0.000$

considering the number of persons in a household, women from households with $3-5$ persons were $50 \%$ less likely to experience unintended pregnancy compared to women from households with 1-2 persons. Women in non-slum settlements were about 2.2 times $(\mathrm{p}<0.002)$ more likely to experience unintended pregnancy than the women living slum settlements.

The results of Model II show that women in formal employment were $55 \%(\mathrm{p}<0.01)$ less likely to experience unintended pregnancy compared to those who were either unemployed or students. Luhya and Luo women also remained more likely to experience unintended pregnancy compared to their Kikuyu counterparts. Non- 
slum women were still 1.8 times more likely to experience unintended pregnancy compared to those from slum settlements. Women who were at least 20 years old were less likely to experience unintended pregnancy compared to those aged 15-19 years. Considering marital status, currently married and formerly married women were less likely to experience unintended pregnancy compared to those who were never married.

Further analysis of the determinants of unintended pregnancy in each of the settlements was conducted. The results for slum women are presented in Table 4 and those of non-slum women in Table 5 .

The results show that in both types of settlements, ethnicity and marital status have each statistically significant effect on unintended pregnancy. In both settlements, single

Table 4 Odds ratio, based on logistic regression analysis, of unintended pregnancy among women, 15-49 in Slum settlements in Nairobi Kenya

\begin{tabular}{|c|c|c|c|c|}
\hline \multirow[t]{2}{*}{ Characteristic } & \multicolumn{2}{|c|}{ Model I } & \multicolumn{2}{|c|}{ Model II } \\
\hline & OR & $p$ & OR & $p$ \\
\hline \multicolumn{5}{|l|}{ Education [Ref: None] } \\
\hline Primary & 1.71 & 0.301 & 1.59 & 0.390 \\
\hline Secondary & 1.74 & 0.295 & 1.54 & 0.441 \\
\hline Higher & 3.42 & 0.106 & 1.50 & 0.640 \\
\hline \multicolumn{5}{|c|}{ Wealth index [Ref: Poor] } \\
\hline Medium & 1.00 & 0.989 & 1.02 & 0.938 \\
\hline Rich & 0.62 & 0.046 & 0.70 & 0.150 \\
\hline \multicolumn{5}{|c|}{ Employment status [Ref: Unemployed/student] } \\
\hline Informal employment & 0.99 & 0.957 & 0.97 & 0.930 \\
\hline Formal employment & 0.73 & 0.422 & 0.77 & 0.527 \\
\hline Self employed & 0.92 & 0.713 & 1.00 & 0.998 \\
\hline \multicolumn{5}{|l|}{ Ethnicity [Ref: Kikuyu] } \\
\hline Luhya & 1.65 & 0.064 & 2.21 & 0.007 \\
\hline Luo & 1.61 & 0.094 & 1.83 & 0.049 \\
\hline Kamba & 0.61 & 0.073 & 0.76 & 0.360 \\
\hline Other & 0.77 & 0.442 & 1.09 & 0.809 \\
\hline \multicolumn{5}{|c|}{ Household size [Ref: 1-2 persons] } \\
\hline 3-5 persons & 0.41 & 0.000 & 0.60 & 0.065 \\
\hline $6+$ persons & 0.77 & 0.331 & 1.06 & 0.862 \\
\hline \multicolumn{5}{|l|}{ Age [Ref: 15-19] } \\
\hline $20-34$ & & & 0.20 & 0.030 \\
\hline $35-49$ & & & 0.19 & 0.031 \\
\hline \multicolumn{5}{|l|}{ Parity [Ref: 0] } \\
\hline 1-2 children & & & 2.40 & 0.000 \\
\hline $3+$ children & & & 2.45 & 0.000 \\
\hline \multicolumn{5}{|c|}{ Marital status [Ref: Never married] } \\
\hline Currently married & & & 0.12 & 0.000 \\
\hline Formerly married & & & 0.26 & 0.000 \\
\hline
\end{tabular}

Table 5 Odds ratio, based on logistic regression analysis, of unintended pregnancy among women, 15-49 in Non-Slum settlements in Nairobi Kenya

\begin{tabular}{lccccc}
\hline Characteristic & Model I & & \multicolumn{2}{c}{ Model II } \\
\cline { 2 - 5 } & OR & $p$ & OR & $p$ \\
\hline
\end{tabular}

Education [Ref: None]

$\begin{array}{lllll}\text { Primary } & 0.62 & 0.757 & 0.26 & 0.381 \\ \text { Secondary } & 0.50 & 0.657 & 0.21 & 0.299 \\ \text { Higher } & 0.54 & 0.692 & 0.18 & 0.252 \\ \text { Wealth index [Ref: Poor] } & & & & \\ \text { Medium } & 0.96 & 0.869 & 1.09 & 0.757 \\ \text { Rich } & 0.75 & 0.280 & 0.86 & 0.614\end{array}$

Employment status [Ref: Unemployed/student]

$\begin{array}{lllll}\text { Informal employment } & 1.10 & 0.803 & 0.91 & 0.844 \\ \text { Formal employment } & 0.39 & 0.001 & 0.44 & 0.010 \\ \text { Self employed } & 0.51 & 0.008 & 0.62 & 0.112\end{array}$

Ethnicity [Ref: Kikuyu]

$\begin{array}{lllll}\text { Luhya } & 1.49 & 0.168 & 1.84 & 0.069 \\ \text { Luo } & 2.12 & 0.009 & 2.54 & 0.006 \\ \text { Kamba } & 1.90 & 0.050 & 3.31 & 0.002 \\ \text { Other } & 0.80 & 0.590 & 1.32 & 0.553\end{array}$

Household size [Ref: 1-2 persons]

3-5 persons

$\begin{array}{llll}0.92 & 0.882 & 1.45 & 0.569\end{array}$

$6+$ persons

$\begin{array}{llll}1.03 & 0.949 & 1.25 & 0.732\end{array}$

Age [Ref: 15-19]

20-34

$0.86 \quad 0.898$

$35-49$

$0.85 \quad 0.890$

Parity [Ref: 0]

$\begin{array}{lll}1-2 \text { children } & 0.62 & 0.536\end{array}$

$3+$ children $\quad 0.95 \quad 0.945$

Marital status [Ref: Never married]

$\begin{array}{llll}\text { Currently married } & 0.06 & 0.000\end{array}$

$\begin{array}{lll}\text { Formerly married } & 0.15 & 0.000\end{array}$

women were significantly more likely to experience unintended pregnancy than their currently married or formerly married counterparts. Similarly, in both settlements, being Luo or Luhya was associated with a higher likelihood of experiencing unintended pregnancy compared to being a Kikuyu. Parity and age are the other factors that have statistically significant effects in the slum settlements only. In the slum settlements, the likelihood of experiencing unintended pregnancy increased with parity. For example, women of parity 1-2 children and those of at least parity 3 were 2.4 and 2.5 times, respectively, as likely as women of zero parity to experience unintended pregnancy. In contrast, the likelihood of experiencing unintended pregnancy among the women in the slum settlements declined with the age of the woman. Young women (15-19) are 
significantly more likely to experience unintended pregnancy compared to older women (20-34 or 35-49).

\section{Discussion}

This study addressed the prevalence, socio-economic and demographic correlates of unintended pregnancy among slum and non-slum women in Nairobi. About $24 \%$ of the women reported an unintended pregnancy. The 2008-09 KDHS indicates that 43\% of Kenyan women and $29.4 \%$ of women in Nairobi Province in particular reported their most recent pregnancies to be unintended. Judging by these figures, the incidence of unintended pregnancy among women in the study was slightly lower than the national and provincial averages. While many factors can explain the comparatively lower incidence of unintended pregnancy in the study communities, the growing availability of family planning products in the study communities needs to be highlighted. The study areas have also been the targets of aggressive family planning campaigns in recent times [23].

In the study, education and household wealth were surprisingly not strongly associated with unintended pregnancy. This is contrary to many other studies on the correlates of unintended pregnancy $[24,25]$. However, judging by results of the logistic regression, it is difficult to entirely dismiss the influence of income and educational levels on unintended pregnancies among the women we studied. This is because employment status was a strong predictor of unintended pregnancy. Women in formal employment were also $55 \%(\mathrm{p}<0.01)$ less likely to experience unintended pregnancy compared to unemployed women or students. Formal employment is related in critically important ways to earning capacity, educational levels, and social networks [26-29]. Women in formal employment tend to earn more, have higher levels of reproductive health knowledge and participate in social networks that support family planning and reduced fertility [29-34].

Further, similar to previous work from Kenya, we found that young and unmarried women in the study were, irrespective of their educational attainment and household wealth status, at high risk of experiencing unintended pregnancy $[21,22,35,36]$. Research in Kenya generally, and in the specific areas that we covered indicates that young people have limited access to quality sexual and reproductive health information, including knowledge on contraception $[5,6,19,21,22,36]$. In addition to the unaffordability of family planning products and services to many girls, several hospitals and clinics in Kenya where family products and services - mainly, oral contraceptives, injectables, implants, male condoms, IUDs and, very rarely, vasectomy - are subsidized or provided free of charge regularly experience stock outs and a dearth of qualified providers [37]. Stigma, inadequate sexuality information and cultural pressure to appear sexually chaste and inexperienced also hinder utilization of family planning services among young girls and unmarried girls [38]. Most young girls in Kenya also rely on parents, equally uninformed peers, and popular media for information on sexual maturation, pregnancy and sexuality, who often do not provide them correct and timely information, exposing them to sexual and reproductive health risks including unintended pregnancies $[21,36,39,40]$.

Interestingly too, ethnicity had statistically significant effect on unintended pregnancy. In both settlements, being Luo or Luhya was associated with a higher likelihood of experienced unintended pregnancy compared to being a Kikuyu. Due largely to their growing access to higher education, greater participation in paid employment and widespread use of contraceptives, the Kikuyu as a whole has continued to experience steadydeclines in fertility in the recent past. On the other hand, while there is evidence of higher levels of pronatalism among the Luyha and Luo than among the Kikuyu, there is also research suggesting that women from the former ethnic groups are among the poorest and least empowered in Kenya [41-44]. The rates of unintended pregnancies from the Provinces where the Luo and Luyha traditional reside, e.g., Western and Nyanza Provinces are also among the highest in Kenya [5,19]. Higher levels of unintended pregnancy among the Luo and Luyha in the study may relate to their lower empowerment status, poorer socioeconomic status and more limited access to family planning products $[21,45,46]$. Compared to Kikuyu women, more Luo and Luhya women in Kenya currently survive as dependents of men in relationships that hinder their power to protect themselves from unintended pregnancies and sexually transmitted infections $[21,47,48]$. The poorer socio-cultural and economic status of Luo and Luyha women finds expression, among other things, in the ideology of patrilineal inheritance, male-biased property rights, virilocalism and male ownership of children. For instance, among the Luo, upon the death of a husband, his widow(s) is also eventually claimed as property by brothers-in-laws or other male relatives of the deceased husband [21]. On the other hand, in the Kikuyu culture, children and the responsibility of catering for them are primarily perceived as women's, not men's. Kikuyu women may thus be much keener in looking for ways to avoid pregnancies that they are not well-prepared for $[49,50]$.

\section{Conclusion}

The study found a high prevalence of unintended pregnancy among the study population. Young and unmarried women, irrespective of their educational attainment and household wealth status, had a higher likelihood of experiencing unintended pregnancy. Except for the results on educational attainments and household wealth, 
our findings compared with the results reported in the literature.

The study results are critical and raise the need for programs and strategies to improve access to contraceptive services and related education, socio-economic status and information and communication particularly among the young and unmarried women. Increased availability and accessibility of family planning services is crucial to reducing unintended pregnancy among the study population. Concerted and coordinated multisectoral efforts are key to expanding access and uptake of family planning services. Increased access should be accompanied with improvement in the quality of care and availability of information about effective utilization of family planning methods.

\section{Competing interests}

The authors have no competing interests.

\section{Authors' contribution}

LI: Conceptualized the paper and led the literature review. Cl: Conceived and led the study project and wrote sections of the manuscript. RO: conducted data analysis, wrote the results section and reviewed drafts of the manuscript. All authors read and approved the final manuscript.

\section{Acknowledgments}

This study was supported by the Consortium for Research on Unsafe Abortion, with grants from DfID. We also acknowledge support from the STEP UP (Strengthening Evidence for Programming on Unintended Pregnancy) Research Programme Consortium, funded by UK aid from the UK Government (Grant SR1109D-6) and the E2A project supported by USAID. Core support to APHRC by the Rockefeller Foundation, SIDA and the William and Flora Hewlett Foundation supported APHRC staff to develop the study and contribute to this paper. Authors are grateful to the study respondents and research assistants.

\section{Author details}

'Population Studies and Research Institute, University of Nairobi, P.O.BOX 30197, 00100, GPO, Nairobi, Kenya. ${ }^{2}$ African Population and Research Centre (APHRC), P.O.BOX 10787, 00100, Nairobi, Kenya. ${ }^{3}$ Population Services International (Formerly worked with APHRC), P.O.BOX 22591-00400, Nairobi, Kenya.

Received: 10 October 2012 Accepted: 4 March 2013 Published: 19 March 2013

\section{References}

1. Bitto A, Gray RH, Simpson Jl, Queenan JT, Kambic RT, Perez A, Mena P, Barbato $M, L i C$, Jennings V: Adverse outcomes of planned and mistimed pregnancies among users of natural family planning: a prospective study. Am J Public Health 1997, 97(3):338-343.

2. Eggleston E: Determinants of unintended pregnancy among women in Ecuador. IntFam Plan Perspect 1999, 25(1):27-33.

3. Gage AJ: Does fertility timing influence the utilization of maternal health care services? Evidence from Kenya and Namibia. Working papers in African Demography, University Park; 1996. Population Research Institute, Pennsylvania State University, No. AD96-05.

4. Joyce TJ, Grossman M: Pregnancy wantedness and early initiation of prenatal care. Demography 1990, 27(1):1-17.

5. Magadi M: Poor pregnancy outcomes among adolescents in south Nyanza region of Kenya. Afr J Reprod Health 2006, 10(1):26-38.

6. Forrest JD: Epidemiology of unintended pregnancy and contraceptive use. Am J Obstetrics Gynaecology 1994, 170(5):1485-1489.

7. Adetunji J: Levels, trends and determinants of unintended childbearing in developing countries.DHS Analytical Report No.8. Calverton, Maryland: Macro International Inc; 1998
8. Central Bureau of Statistics (CBS) [Kenya], Ministry of Health (MOH [Kenya], and ORC Macro: Kenya Demographic and Health Survey 2003. Calverton, Maryland: CBS, MOH and ORC Macro; 2004.

9. Kenya National Bureau of Statistics (KNBS) and ICF Macro: Kenya demographic and health survey, 2008-09. Calverton, Maryland: KNBS and ICF Macro; 2010.

10. Centre for the Study of Adolescence: Down the drain: counting the cost of teenage pregnancy and school drop Out in Kenya. CSA: Nairobi: The centre for study of adolescence; 2008.

11. Bongaarts J: Trends in unwanted childbearing in the Developing World. Stud Fam Plan 1997, 28(4):267-277.

12. Adhikari R: KusolSoonthorndhada. PramotePrasartkul: Correlates of unintended preganncy among currently married women in Nepal.BMC Internaltional Health and Human Rights; 2009. doi:10.1186/142-698x-9-17.

13. Shaheen AA, Diaaeldin M, Chaaya M, ElRooeiheb Z: 'Unintended pregnancy in Egypt: evidence from the national study on women giving birth in 1999'. East Mediterr Health J 2007, 13(6):1392-1404.

14. Casterline JB, El-Zanatay F, El-Zeini LO: Unmet need and unintended fertility: longitudinal evidence from Upper Egypt. Int Fam Plan Perspect 2003, 29(4):158-166.

15. Herold JM, Thompson NJ, Velenzuela MS, Morris L: Pregnancy and sex education in Chile: a behavioral model. J Biosocial Sci 1994, 26(4):427-439.

16. Mbizvo MT, et al: Unplanned pregnancies in Harare: what are the social and sexual determinants? SocSci Med 1997, 45(6):937-942.

17. Kost K, Forrest JD: Intentions status of US births in 1988: differences by mother's socio demographic characteristics. FamPlannPerspect 1995, 27(1):11-17.

18. Sulak PJ, Haney AF: Unwanted pregnancies: understanding contraceptive use and benefits in adolescents and older women. Am J ObstetGynecol 1993, 168(6):2042-2048.

19. Williams $L$ : Determinants of unwanted childbearing among ever married women in the United States 1973-1988. FamPlannPerspect 1991, 23(5): 212-221.

20. Magadi MA: Unplanned childbearing in Kenya: the socio-demographic correlates and the extent of repeatability among women. SocSci Med 2003, 56(1):167-178.

21. Izugbara CO, Ochako R, Izugbara C: Gender scripts and unwanted pregnancy among urban Kenyan women. Cult Health Sex 2011, 13(9): 1031-1045.

22. African Population and Health Research Centre: Population and health dynamics in Nairobi informal settlements. Nairobi: African Population and Health Research Centre; 2002.

23. African Population Health Research Centre: Population and health in the slums of Nairobi. African Population and Health Research Centre (APHRC): Nairobi; 2002.

24. Finer LB, Henshaw SK: Disparities in rates of unintended pregnancy in the United States, 1994 and 2001. Perspect Sex Reprod Health 2006, 38(2):90-96.

25. Finer LB, Zolna MR: Unintended pregnancy in the United States: incidence and disparities, 2006. Contraception 2011, 84(5):478-485.

26. Heemskerk M: Self-employment and poverty alleviation: Women's work in artisanal gold mines. Hum Organ 2003, 62(1):62-73.

27. Goldin C: The quiet revolution that transformed women's employment, education, and family.National Bureau of Economic Research (NBER). Cambridge, Massachusett, USA: NBER Working Paper No.11953; 2006.

28. Al Riyami A, Afifi M, Mabry RM: Women's autonomy, education and employment in Oman and their influence on contraceptive use. Reprod Health Matters 2004, 12(23):144-154

29. Behrman JR, Kohler HP, Watkins SC: Social networks and changes in contraceptive use over time: evidence from a longitudinal study in rural kenya. Demography 2002, 39(4):713-738.

30. Shapiro D, Tambashe BO: The impact of women's employment and education on contraceptive use and abortion in Kinshasa, Zaire. Stud Fam Plan 1994, 25(2):96-110. Population Council.

31. Salaff JW, Greve A: Can women's social networks migrate?. Women's Studies International Forum. June-July 2004, 27(2):149-162.

32. Willis K: Women's work and social network use in Oaxaca City, Mexico. Bull Lat Am Res 1993, 12(1):65-82.

33. Hindin MJ: Women's autonomy, women's status and fertility-related behavior in Zimbabwe. Population Res Policy Review no 2000, 19(3):255-282.

34. Hogan DP, Berhanu B, Hailemariam A: Household organization, women's autonomy, and contraceptive behavior in southern Ethiopia. Stud FamPlann 2008, 30(4):302-314. 
35. Nzioka C: Perspectives of adolescent boys on the risks of unwanted pregnancy and sexually transmitted infections: Kenya. Reprod Health Matters 2001, 9(17):108-117. doi:10.1016/50968-8080(01)90014-x.

36. Crichton J, Okal J, Kabiru CW, Zulu EM: Emotional and psychosocial aspects of menstrual poverty in resource-poor settings: a qualitative study of the experiences of adolescent girls in an informal settlement in Nairobi. Health Care for Women International; 2012. doi:10.1080/70399332.2012.740112.

37. Agwanda A: "Reproductive health in kenya: an examination of safe motherhood initiative in light of the millennium development goals.", Reproductive, maternal and child health in Africa. Dakar Senegal: Current developments and future directions; 2006. Union of African Studies TRN No 6: 149-75.

38. Aloo-Abunga C: Country Analysis of Family Planning and HIV/AIDS, Kenya. Washington, DC: Policy Project; 2003.

39. Crichton J, Ibisomi L, Gyimah SO: Mother-daughter communication about sexual maturation, abstinence and unintended pregnancy: experiences from an informal settlement in nairobi, kenya. J Adolesc 2012, 35(1):21-30.

40. Izugbara CO, Otsola K, Ezeh AC: Men, women, and abortion in Central Kenya: a study of lay narratives. Medical Anthropology no 2009, 28(4):397-425.

41. Place $F$, Adato $M$, Hebinck $P$ : Understanding rural poverty and investment in agriculture: an assessment of integrated quantitative and qualitative research in western kenya. World Dev 2007, 35(2):312-325.

42. Cattell MG: "Holding up the sky: gender, age and work among the Abaluyia of Kenya.". In Ageing in Africa: Sociolinguistic and anthropological approaches. Edited by Makoni S, Straoeken S. Aldertshort: Ashgate; 2002:157-177.

43. Dodoo F, Tempenis M: Gender, power, and reproduction: rural--urban differences in the relationship between fertility goals and contraceptive Use in kenya*. Rural Sociol 2002, 67(1):46-70.

44. Fotso JC, Ezeh A, Madise N, Ziraba A, Ogollah R: What does access to maternal care mean among the urban poor? Factors associated with use of appropriate maternal health services in the slum settlements of Nairobi, Kenya. Matern Child Health J 2009, 13(1):130-137.

45. Watkins SC: Local and foreign models of reproduction in Nyanza Province, Kenya. Population and Development Review no. 2000, 26(4):725-759.

46. Beguy D, Kabiru CW, Zulu EM, Ezeh AC: Timing and sequencing of events marking the transition to adulthood in two informal settlements in Nairobi, Kenya. J Urban Health 2011, 88:318-340.

47. Swallow B, Onyango L, Meinzen-Dick R, Holl N: Dynamics of poverty, livelihoods and property rights in the lower Nyando basin of Kenya, Paper read at International Workshop on Africa Water Laws. Gauteng, South Africa: Pluralistic Frameworks for Rural Water Management in Africa; 2005

48. Gunga SO: The politics of widowhood and Re-marriage among the Luo of kenya. Thought and Practice: A Journal of the Philosophical association of Kenya no 2009, 1(1):165-178.

49. Price N: The changing value of children among the Kikuyu of Central Province Kenya. Africa no 1996, 66(03):411-436.

50. Ciancanelli P: Exchange, reproduction and sex subordination among the Kikuyu of East Africa. Review of Radical Political Economics no 1980, 12(2):25-36.

doi:10.1186/1471-2393-13-69

Cite this article as: Ikamari et al:: Prevalence and determinants of unintended pregnancy among women in Nairobi, Kenya. BMC Pregnancy and Childbirth 2013 13:69.

\section{Submit your next manuscript to BioMed Central and take full advantage of:}

- Convenient online submission

- Thorough peer review

- No space constraints or color figure charges

- Immediate publication on acceptance

- Inclusion in PubMed, CAS, Scopus and Google Scholar

- Research which is freely available for redistribution 